\title{
Positron Emission Tomography Correlations in and Beyond Medial Temporal Lobes
}

\author{
Endel Tulving,* Reza Habib, Lars Nyberg, \\ Martin Lepage, and Anthony R. McIntosh \\ Rotman Research Institute of Baycrest Centre, Toronto,
Canada
}

ABSTRACT This article discusses the potential usefulness of brain/ behavior correlational analyses in functional neuroimaging studies of memory, and how such analyses can illuminate the role of medial temporal lobes (MTL) and the hippocampus in episodic and declarative memory processes such as encoding and retrieval. Reanalysis of the results of four previously reported positron emission tomography (PET) studies yielded evidence of both positive and negative between-subjects correlations between recognition-memory accuracy and regional blood flow. The sites of these correlations were in MTL regions as well as in other cortical and subcortical areas, including frontal lobes (Brodmann areas 6, 9, 10, 11, and 47), temporal lobes (BAs 21, 22, and 38), insula, fusiform gyrus, and cuneus/precuneus. These findings were discussed with respect to issues such as localization of the correlation sites, the distinction between brain sites revealed by brain/cognition correlational analyses ("how" sites) and those yielded by cognitive subtraction methods ("what" sites), the tendency of the "how" sites in MTL to occur in the left hemisphere, the tendency of other "how" sites to occur in one or the other hemisphere, rather than bilaterally, and the meaning and "reality" of both brain/behavior correlations and task-related activations. Because of the known incidence of false-positives, all neuroimaging data, including those involving the localization of "what" and "how" memory sites in MTL and other brain regions, need to be interpreted cautiously, and findings of individual studies should not be overinterpreted. Hippocampus 1999;9:71-82. 단. 1999 Wiley-Liss, Inc.

KEY WO RDS: hippocampus; PET; neuroimaging; retrieval performance; false-positives

Despite the widely held belief that functional neuroimaging studies of memory have failed to capture the hippocampus and medial temporal lobes (M TL) in their net (Fletcher et al., 1995b; Kopelman et al., 1998), and occasional complaints that they have had only limited success in throwing light on the role of these structures in memory (Fletcher et al., 1997), positron emission tomography (PET) and functional magnetic resonance imaging (FM RI) research on "hippocampal memory" is flourishing. The Special Issue of $\mathrm{H}$ ippocampus provides living testimony to this fact.

\section{WHAT HAS BEEN AND CAN BE LEARNED?}

The central issue here is what we have learned, and what we can learn, about the role of the hippocampus and M TL in those forms of memory for

Grant sponsor: Anne and Max Tanenbaum Foundation; Grant sponsor: $\mathrm{N}$ atural Sciences and Engineering Research Council of Canada.

*Correspondence to: Endel Tulving, Rotman Research Inst. of Baycrest Centre, 3560 Bathurst Street, Toronto O N M 6A 2E1, Canada.

Accepted for publication 300 ctober 1998 which its relevance is well established, episodic (Tulving, 1983) and declarative (Squire, 1992). An appropriately neutral query could be put as follows: W hat do we know now, some half dozen years after the publication of the first "true" functional imaging study of memory (Squire et al., 1992), and not neglecting data from more traditional lesion analyses, that we did not know before and that is worth knowing about what the hippocampus and M TL "do" in or for memory?

Based on the findings of numerous studies, suggestions have been offered as to the nature of the "role" of M T L in human memory. Some of these have been rather general, others more specific.

The more general suggestions usually, but not necessarily, are meant to apply to the hippocampus or M TL bilaterally. They include the following: H ippocampus plays a role in memory for past events ( $M$ attioli et al., 1996). H ippocampal activation signals episodic retrieval of previously studied objects (Schacter et al., 1995). MTL regions are more active during early stages of memorization of abstract designs than later stages (Petersson et al., 1997). H ippocampus has been claimed to be involved in the detection and assessment of novelty of incoming information (Tulving et al., 1994c, 1996; Stern et al., 1996; Knight, 1996; D olan and Fletcher, 1997; Parkin, 1997; G runwald et al., 1998). H ippocampus and adjacent cortex participate in encoding of information about faces but not about its retrieval (H axby et al., 1996).

0 ther suggestions include specific references to one or the other hemisphere. They include the following. M edial temporal lobe regions in the two hemispheres are engaged automatically and in material-specific ways at the time of perception of objects and their encoding into memory (M artin et al., 1997). Left hippocampus shows greater activation in retrieval of deeply encoded than in retrieval of shallowly encoded words (Rugg et al., 1997). Parahippocampal regions are activated depending upon task-related response requirements: Left hemisphere regions are involved in nonmatching tasks, and right in matching tasks (Fujii et al., 1997). Right hippocampal regions are involved in processing (recognition) of well-known faces, whereas left hippocampal regions are involved in episodic recognition of faces (Kapur et al., 
1995). Left medial temporal lobe is associated with encoding of words, right temporal lobe with encoding of unfamiliar faces (Kelley et al., 1998).

And then there are even stronger assertions, explicit or implied, that state not only what the hippocampus and $M T L$ do, but also what they do not do. H ippocampal formation is involved in actual conscious recollection of previously studied events but not in the effort to do so (Schacter et al., 1996; Schacter, 1997). H ippocampus is involved in recall but not in recognition (Aggleton and Saunders, 1997; see also Aggleton and Brown, in press). H ippocampal formation plays a role in the establishment of associations among components of visual displays, but not in novelty detection, encoding of single items, or retrieval of associations (H enke et al., 1997). H ippocampus shows activity when subjects recognize exactly the same line drawings that they encoded earlier, but not when the size or orientation of the objects are changed at retrieval (Schacter et al., 1997). Right parahippocampal region is activated when subjects passively view "scenes," that is, spaces that are either filled with objects or are empty, but it is not activated when they view "spaceless" objects or faces (Epstein and Kanwisher, 1998), suggesting that this area serves to represent spaces. Right parahippocampal gyrus is activated when subjects view spaces filled with objects, but not activated when they explore empty environment (Maguire et al., 1998; see also M aguire, 1997), suggesting that encoding of objects in space is critical for such activation. Right hippocampus is specifically recruited for mental retrieval of routes through large-scale spatial environments, but not recruited for comparable nontopographical information (M aguire et al., 1996b; see also M aguire et al., 1996a). The hippocampus (along with parietal cortex) mediates retrieval of information about self-centered movement through space, but is less involved in the remembering of spatial layouts as such (Berthoz, 1997).

Finally, there are uncertainties. The issue of material-specificity of the $M T L$ regions in the two hemispheres serves as one example. 1) There is some evidence for material-specific memory deficits attributable to hippocampal damage (Baxendale et al., 1998). 2) There is good evidence, from both FMRI and PET studies, corroborating other data, that the anterior part of the right parahippocampal gyrus is specifically involved in retrieval of information about objects-in-places (M ilner et al., 1997). 3) There is evidence that what has been interpreted as material specificity in the differential consequences of left and right hippocampal damage may be attributable to other factors (Ridley and Baker, 1997; H el mstaedter et al., 1997; D obbins et al., 1998).

This exceedingly compressed overview of some of the findings and conclusions drawn from functional neuroimaging and other studies presents a total picture that is rich, interesting, and challenging. To further titillate the observer of the scene, two studies (Lepage et al., 1998; Schacter and Wagner, 1999, this issue) have reported summarized data garnered from many studies, with intriguing results.

$M$ eta-analyses of both published and unpublished PET data have shown that encoding activations tend to occur more frequently in the rostral (anterior) than the caudal (posterior) regions of $M T L$, whereas FM RI data show the opposite tendency (see Schacter and Wagner, this issue, for review). These metaanalyses are based on a relatively large number of studies. Lepage et al. (1998) reported 54 activation points in the M TL originating from 20 studies representing 32 task comparisons. Schacter and Wagner (1999) examined PET and FMRI studies separately. Their review of PET hippocampal activations was based on a survey of 33 different studies in which 52 task comparisons yielded $81 \mathrm{MTL}$ activation points. Their review of FMRI hippocampal activations was based on a survey of nine studies in which 17 task comparisons yielded $31 \mathrm{MTL}$ activations. At the same time, PET-based retrieval activations in MTL show a reversed gradient to that of PET-based encoding activations: Retrieval activations tend to be concentrated more in the caudal than in the rostral regions.

\section{GOODNESS OF MEMORY PERFORMANCE}

We have presented this quick overview of functional imaging and some other, related findings concerning hippocampal memory to make two points: 1) The data reported in all these studies should help to dispel the notion that functional neuroimaging is too feeble a technique to measure hippocampal activity, for whatever reason, and 2) even at this early stage of functional imaging analyses of the role of MTL and the hippocampus in memory, many different ideas have al ready been proposed. Such wealth attests to the fertility of the investigators' minds, but it should also cause concern about the use of the technique. Is it possible that all the ideas already on the table, and others yet to come, are correct? And if not, how should we proceed to sort them out?

The overview also leads to another point, one that is central to the present report. The point is this: All the PET and FM RI studies whose findings or conclusions were summarized above have one important feature in common. In one way or another they all have addressed the same question: Is M TL, or any one of its individual components, including the hippocampus, involved in X, where $X$ stands for an activity or function or behavioral/ cognitive expression? Thus, $X$ might be memory for past events, episodic retrieval of previously studied objects, memorization of abstract designs, detection and assessment of novelty of incoming information, encoding of information about faces, perception of objects and their encoding into memory, processing (recognition) of well-known faces, episodic recognition of faces, conscious recollection of previously studied events, effort to do so, recall of previously studied words, recognition of previously studied words, establishment of associations among components of visual displays, encoding of single items, retrieval of associations, passive viewing of "scenes," passive viewing of "empty" spaces, mental retrieval of routes through large-scale spatial environments, retrieval of nontopographical semantic information, retrieval of information about self-centered movement through space, remembering spatial layouts, and so on, and on, and on. As we saw 
earlier, studies have been done, and results have been reported on the involvement of $M T L$, or the hippocampus, or both, of all of these memory-related activities.

$\mathrm{H}$ ere we report data that speak to a different kind of a role that $M T L$, along with other memory-relevant brain regions, can and probably does play. The question we pose is not whether $M T L$ "does" $X$, but, rather, whether M TL, or some of its components, determines "how well" $X$ is done. Given that, say, some part of the MTL mediates recognition of previously studied words, is it possible that the same part, or some other region in $M T L$, determines how good the recognition performance is? Because functional neuroimaging cannot provide evidence on causation, and can only reveal correlations between brain activity and mental activity, the question has to be toned down a bit: Is it possible that the neuronal activity in some M T L regions systematically covaries with the "goodness" of recognition performance? The question can be posed with respect to the performance of a given individual in different situations (e.g., Kopelman et al., 1998), or to comparative performance of different individuals in a given situation.

$\mathrm{H}$ ere we are concerned with the latter alternative, solely because we had available for analysis relevant data that we had gathered but not analyzed in this fashion in earlier experiments. In examining and evaluating these data, it is important to keep in mind that the results of between-subjects correlational analyses do not predict the results of within-subjects correlational analyses, and vice versa. 0 ur reporting of between-subjects correlational data here does not and should not imply that these data are somehow "superior" to within-subjects covariance analyses. The two kinds can only complement each other, and therefore analyses of both kinds must be conducted.

The issue of between-subjects correlation between brain activity and cognitive performance can be regarded as central to the whole functional neuroimaging enterprise. If we think of the ultimate goal of the enterprise as the mapping of brain activity into mental activity, and vice versa, the search for brain correlates of the goodness (power, efficacy, accuracy) of the individual's performance on a mental task becomes highly relevant. A scenario that right now may seem more like science fiction than science, but which is highly likely to be achieved in the future, is one in which an informed observed can examine an individual's brain map and tell what mental activity theindividual is engaged in, and how well she is performing, and make an equally correct prediction from the mind side to the brain side.

The search for the elements of such a scenario has already begun, and some preliminary data are available. Several studies have been done in which individual performance on memory tests has been related to individual brain activity. For example, in an early study, Grasby et al. (1993a) examined the correlation between blood flow in hippocampal regions and performance on a long-term auditory-verbal memory task. It was found that the number of words correctly recalled correlated with increased blood flow in bilateral hippocampal regions. The spatial extent of the correlation was greater in the left than in the right hemisphere, and the maximal correlation was located in left hippocampus. In addition, significant correlations between memory performance and blood flow were observed in other regions outside the $M T L$, including the posterior hypothalamus, posterior cingulate, the precuneus, and angular gyrus. Eustache et al. (1995) measured the correlation between regional cerebral oxygen consumption and performance on several psychometric tests. A hypothesis-driven search reveal ed significant correlations between the scores on a test of associate learning (recall of paired associates) and metabolism in left thalamus and left hippocampus. A more exploratory statistical analysis additionally revealed significant correlations between right hippocampus metabolism and scores on the associate learning test and a Brown-Peterson D istractor Test. A recent study by Desgranges et al. (1998b) examined correlations between resting cerebral glucoseutilization and performance on a variety of memory tasks. Of particular interest here is that performance on a verbal test (story recall) correlated with glucose utilization in left hippocampus and left posterior cingulate. This was found when a regions-of-interest analysis was conducted and also when a more exploratory, whole-brain search was undertaken with statistical parametric mapping (SPM ).

Some work from our own laboratory has also been concerned with the relation between brain activity and memory performance (N yberg et al., 1996d). Blood flow was measured in six scans while subjects performed three yes/no recognition memory tests of visual words. Two of the scans included words from an incidental study task involving deeper processing, two included words that had been processed more shallowly, and two included non-studied words. When the proportion of correctly recognized words following semantic encoding was cross-correlated with the pattern of whole-brain blood flow across individual subjects, a significant correlation was found in a region in the left anterior M TL. This was so for both scans, suggesting that the correlation was reliable within the sample. Furthermore, activity in this region was significantly correlated with recognition performance following shallow encoding, although the relationship was less strong. No significant correlation was seen between MTL activity and number of yes responses to the non-studied words. To test the general izability of these findings, we also examined the data from an independent study involving yes/no recognition of words following intentional encoding. A significant correlation between activity in left $M T L$, near hippocampus proper, and performance was observed. Activity in other regions than $M T L$ regions also covaried with performance. $D$ ata from the first sample showed strong correlations, following semantic encoding, in cingulate cortex and bilateral inferior prefrontal cortex, and following shallow encoding, in left cerebellum and in bilateral fusiform gyrus.

The purpose of the exercise described here was threefold. The first was to assess the prevalence of PET-based brain/behavior correlations in MTL regions. The second was to examine the extent to which similar correlations would occur in brain regions other than M TL. And third, we were wondering why no negative correlations between memory performance and regional blood flow have been reported (but see the study by D esgranges et al., $1998 \mathrm{~b}$ mentioned earlier). A rethere reall ly no negative correlations in PET "activation" studies? And if there are, what are they like? 
TABLE 1.

Experimental and Performance Measures from the Four Studies Analyzed

\begin{tabular}{llllll}
\hline Study & \multicolumn{1}{c}{$\begin{array}{c}\text { Number } \\
\text { of scans }\end{array}$} & $\begin{array}{c}\text { Type of } \\
\text { stimuli }\end{array}$ & $\begin{array}{c}\text { Type of } \\
\text { test }\end{array}$ & $\begin{array}{c}\text { Behavioral } \\
\text { measure }\end{array}$ & $\begin{array}{c}\text { Mean } \\
\text { performance }\end{array}$ \\
\hline Sentences & 3 New & $\begin{array}{c}\text { Auditory } \\
\text { Sentences } \\
\text { Auditory }\end{array}$ & $\begin{array}{l}\text { Yes/No Recognition } \\
\text { Sentences }\end{array}$ & Accuracy & 1.28 \\
Pictures & 3 Old & Pictures & Yes/No Recognition & Accuracy & 1.42 \\
& 2 New & Pictures & Yes/No Recognition & Accuracy & 2.63 \\
Cross-Modality & 2 Item Recognition & Words & Yes/No Recognition & Hit Rate & 0.85 \\
Same-Modality & 2 Shallow Recognition & Words & Yes/No Recognition & Hit Rate & 0.31 \\
& 2 Deep Recognition & Words & Yes/No Recognition & Hit Rate & 0.58 \\
\hline
\end{tabular}

We obtained the data to explore these issues from four previously done, and at least partially reported, PET studies of episodic encoding and retrieval from our laboratory (Tulving et al., 1994b,c; N yberg et al., 1995, 1996d). For the purpose of the exercise we recalculated and reanalyzed the data from all four studies using a multivariate approach that allowed us to examine consistent brain/behavior correlations (henceforth simply "correlations") across different PET scans of individual studies. Search for such consistency is critical in functional neuroimaging studies, because the possibility of error, including that of false-positivesdetecting sites of brain activations or brain/behavior correlations that are not "real" - can be high. We will return to this problem in the discussion.

\section{PET CORRELATIONS}

We reanalyzed data from four PET studies of recognition memory. Subject and experimental parameters of each study are summarized in Table 1. Common to the four studies was the fact that they included more than one scan during which blood flow was measured while subjects made binary decisions regarding the status of presented items (novel/familiar). The repetition of conditions within studies allowed us to take into account within-study consistencies in brain/behavior correlations, and the use of a similar behavioral procedure across studies made possible the assessment of consistencies between studies. Importantly, though, as outlined below and summarized in Table 1, there were several procedural variations between studies, including differences in terms of material (sentences, pictures, words), compatibility of study-test modality (same, different), and differences in the length of the retention interval (ranging from a few minutes to 24 hours). To the extent that any of these variables affected the specific neural constellations that are engaged during execution of the task, study-specific activation patterns would be observed. These may or may not translate into study-specific correlational patterns.
The first study (sentences) examined the neural mechanisms of auditory sentence recognition in 12 young right-handed subjects (Tulving et al., 1994b). In this study, subjects heard a series of auditory sentences presented to them 24 hours prior to scanning. On the following day while being scanned, subjects were required to discriminate sentences heard on the previous day from new sentences. Six recognition scans were administered. In three scans, subjects heard mainly new sentences and were required to mentally count the number of old sentences, whereas in the remaining three scans subjects heard mainly old sentences and were required to mentally count the number of new sentences. Subjects reported their count following completion of the scan. Performance was scored as the absolute deviation of subjects count of either new or old items from the correct total. This performance variable measures absolute recognition accuracy because false alarms ("old" responses to new items) and misses ("new" responses to old items) are considered equivalent as errors.

The second study (pictures) explored blood flow during picture recognition in 12 young right-handed subjects (Tulving et al., 1994c). The design of this study was similar to the sentences study with the following exceptions: 1) the stimuli were scenic pictures from N ational Geographic, and 2) subjects received two scans of mainly old pictures and two scans of mainly new pictures. Recognition performance was scored in the same manner as for Sentences.

The third study (cross-modality) examined blood flow during visual recognition of previously studied auditory words ( $N$ yberg et al., 1995). Prior to scanning, 11 young right-handed subjects studied words under either semantic or non-semantic encoding conditions. During semantic encoding, subjects decided whether words represented living or non-living things. During nonsemantic encoding, subjects decided whether the speaker was male or female. In different scans, blood flow was measured while subjects recognized semantically or non-semantically encoded words. Two scans of each type of recognition condition were performed. The performance measure was the number of previously presented items subjects recognized (hit rate). 
The fourth study (same-modality) examined recognition of previously presented visual words (N yberg et al., 1996d). Twelve right-handed subjects studied words approximately 10 minutes prior to the recognition scans. In one experimental condition, they received another encoding opportunity 5 minutes later (i.e., 5 minutes prior to the scan). During these two recognition conditions, blood flow was measured while subjects attempted to discriminate previously studied words from new words. Performance was measured in terms of correct recognition of previously presented words.

In each study, all subjects' images were aligned to their first image, spatially transformed into the standard stereotaxic atlas space of Talairach and Tournoux (1988), and smoothed by means of a 10-mm isotropic Gaussian filter using Statistical Parametric $M$ apping software [SPM 96, Wellcome D epartment of Cognitive N eurology, London (Friston et al., 1995)].

Each study was analyzed with the method of Partial Least Squares (M clntosh et al., 1996; Schreurs et al., 1997). Partial Least Squares (PLS) is a multivariate approach that examines the relation between a set of exogenous measures such as experimental design, or subjects' performance measures, and a set of functional brain images. The outcome of PLS is a new set of images (the singular images) that identifies networks of brain regions that optimally relate to particular aspects of the experimental design to a measure of performance. The strength of this relation can be evaluated at the level of the entire image, or at the level of individual voxels, through assessment the contribution of the voxel to the singular image.

In the present case, separate PLS analyses were conducted for each of the four studies. Each analysis examined the image-wide relationship between blood flow and memory performance across all scans of the experiment simultaneously and produced a series of images, which represent the optimal relationship between blood flow and performance at each point in the brain. In each analysis, the image which accounted for the largest proportion of the covariance between blood flow and retrieval performance represented common correlations between blood flow and behavior across all conditions of the study.

The reliability of the correlated regions was assessed by means of a bootstrap procedure. In bootstrapping, subjects are randomly selected into the analysis with replacement from the entire group of subjects within each study. For each new sample, the entirePLS procedure is re-calculated.

This sampling and analysis procedure was carried out 100 times in each study, resulting in estimates of the standard error of the covariance between the singular image and memory performance, and the standard error of the contribution of each voxel to the singular image (which is proportional to its covariance with performance). The ratio of the covariance to its standard error is similar to a z-score, and for the present results, we thresholded the bootstrap results at a ratio of 2.24, approximately equivalent to $\mathrm{P}<.025$ on a two-tailed normal distribution. We focused on the reliability of the voxel contributions to this pattern, rather than a singular image, to determine whether there were certain regions that showed a replicable contribution to memory performance across studies. By using bootstrapping, we emphasize the reliabil- ity of the correlation rather than its magnitude alone. Bootstrapping assesses the reliability of correlations by guarding against the effects of outlier observations. In addition, we thresholded the spatial extent of the activations to those correlation regions greater than 15 voxels in size.

The locations of peak positive correlations between $\mathrm{rCBF}$ and recognition performance are presented in Table 2 , organized by the brain structures in which these correlations were observed in. Table 3 presents the location of peak negative correlations.

Positive MTL correlations were observed in each of the four studies, although in the same-modality study, the bootstrap ratio of the M TL correlation (0.034) fell below the originally specified threshold level. Except for the cross-modal study, all MTL correlations occurred in the left hemisphere; in the cross-modal study, the correlation was bilateral. O nly three other brain regions were correlated in more than one study. These included the fusiform gyrus, cuneus/precuneus, and Brodmann area 6. The remaining correlations were study-specific (see Table 2), and indeed may not be reliable, as we discuss later.

The most prominent sites of negative correlations between $\mathrm{rCBF}$ and recognition performance occurred in the frontal and temporal lobes. Frontal regions included Brodmann areas 9 and 10 bilaterally and Brodmann areas 11 and 47 in the right hemisphere. Temporal regions included the middle temporal gyrus (BA 21) bilaterally and Brodmann areas 22 and 38 in the left hemisphere. The insular region also correlated negatively in three of the four studies examined. With the exception of the cuneus/precuneus region (Sentences and Pictures study), the remaining negative correlations were study specific (seeTable 3 ).

\section{PERFORMANCE CORRELATIONS IN FUNCTIONAL NEUROIMAGING}

The purpose of the meta-analysis whose results are reported here was to examine the possibility that behavioral performance in recognition memory is systematically related to neuronal activity, as reflected in changes in blood flow, not only in medial temporal lobe regions but also in other regions of the brain. We went beyond previous reports in three ways: 1) Considering data from a small set of four studies instead of a single study, 2) not focusing solely on MTL but treating it as a (key) component of more widely distributed memory circuits of the brain, and 3) dealing with and reporting both positive and negative brain/behavior correlations.

The data we describe serve primarily to point to the potential usefulness of the method of between-subjects correlations in neuroimaging studies of memory, as well as other higher cognitive processes. Because the four studies whose data we examined were initially designed to maximize the performance on the tasks of interest, and thereby minimize between-subject variability, the fact that anything useful came out of the performance-related analysis is both surprising and promising. It suggests that under more optimal conditions, including planned enhancement of 
TABLE 2.

Regions of Peak Positive Correlations Between Recognition Performance and rCBF

\begin{tabular}{|c|c|c|c|c|c|c|}
\hline Study & $X$ & $\mathrm{Y}$ & $\mathrm{Z}$ & Size & $\begin{array}{c}\text { Bootstrap } \\
\text { ratio }\end{array}$ & Structure \\
\hline \multicolumn{7}{|c|}{ Medial Temporal Lobe } \\
\hline Sentences & -28 & -24 & -8 & 88 & 2.91 & Hippocampus \\
\hline Pictures & -32 & -32 & -4 & 42 & 2.72 & Hippocampus \\
\hline \multirow[t]{2}{*}{ Cross-Modality } & 28 & -14 & -28 & 15 & 2.74 & Parahippocampal Gyrus \\
\hline & -26 & 2 & -12 & 131 & 3.13 & Amygdala \\
\hline Same-Modality* & -16 & -26 & -4 & 18 & 2.11 & Parahippocampal Gyrus \\
\hline \multicolumn{7}{|l|}{ Fusiform Gyrus } \\
\hline Sentences & 32 & -36 & -16 & 22 & 2.50 & Fusiform Gyrus \\
\hline Pictures & 34 & -68 & 0 & 116 & 2.87 & Fusiform Gyrus \\
\hline \multicolumn{7}{|l|}{ Frontal Gyri } \\
\hline Sentences & -28 & 0 & 44 & 42 & 2.85 & Middle Frontal Gyrus (BA 6) \\
\hline Pictures & -16 & 34 & 28 & 27 & 2.44 & Medial Frontal Gyrus (BA 9) \\
\hline Cross-Modality & 32 & 40 & -8 & 24 & 2.40 & Inferior Frontal Gyrus (BA 47) \\
\hline Same-Modality & -20 & -2 & 48 & 255 & 2.52 & Medial Frontal Gyrus (BA 6) \\
\hline \multicolumn{7}{|l|}{ Cingulate Gyrus } \\
\hline \multirow[t]{3}{*}{ Pictures } & 18 & 38 & 4 & 42 & 2.48 & Cingulate Gyrus (BA 24/32) \\
\hline & 12 & 16 & 24 & 19 & 2.45 & Cingulate Gyrus (BA 24) \\
\hline & -2 & -12 & 36 & 57 & 2.83 & Cingulate Gyrus (BA 24) \\
\hline \multicolumn{7}{|c|}{ Cuneus/Precuneus } \\
\hline Pictures & -14 & -82 & 8 & 43 & 2.68 & Cuneus (BA 17) \\
\hline Same-Modality & -8 & -86 & 40 & 124 & 2.48 & Cuneus (BA 19) \\
\hline \multicolumn{7}{|c|}{ Occipital/Lingual Gyrus } \\
\hline \multirow[t]{2}{*}{ Pictures } & -30 & -74 & 8 & 45 & 2.73 & Middle Occipital Gyrus (BA 19) \\
\hline & 22 & -88 & -4 & 42 & 2.60 & Lingual Gyrus (BA 18) \\
\hline \multicolumn{7}{|l|}{ Caudate Nucleus } \\
\hline Pictures & 26 & -32 & 8 & 336 & 2.72 & Caudate Tail \\
\hline \multicolumn{7}{|l|}{ Cerebellum } \\
\hline \multirow[t]{2}{*}{ Cross-Modality } & 32 & -66 & -28 & 47 & 2.50 & Cerebellum \\
\hline & 30 & -42 & -20 & 55 & 2.83 & Cerebellum \\
\hline \multicolumn{7}{|l|}{ Brainstem } \\
\hline \multirow[t]{2}{*}{ Cross-Modality } & 12 & -16 & -20 & 44 & 2.85 & Brainstem \\
\hline & -6 & -10 & -12 & 114 & 2.78 & Brainstem \\
\hline
\end{tabular}

*This coordinate is significant at $P<0.05$.

between-subjects variance of performance, the outcome of correlational analyses might be considerably more decisive.

It is useful to note at this point that our exercise and its outcome says nothing about the sources or causes of the between-subject blood flow differences at the sites of observed correlations. Specifically, it is not known whether these differences are "acute," that is, related to the particular cognitive task, here episodic recognition, or whether they are "chronic," that is, 
TABLE 3.

Regions of Peak Negative Correlations Between Recognition Performance and rCBF

\begin{tabular}{|c|c|c|c|c|c|c|}
\hline Study & $X$ & $\mathrm{Y}$ & Z & Size & $\begin{array}{c}\text { Bootstrap } \\
\text { ratio }\end{array}$ & Structure \\
\hline \multicolumn{7}{|l|}{ Frontal Gyri } \\
\hline \multirow[t]{3}{*}{ Sentences } & -18 & 58 & 12 & 113 & 3.08 & Superior Frontal Gyrus (BA 10) \\
\hline & 18 & 50 & 12 & 158 & 2.65 & Medial Frontal Gyrus (BA 10) \\
\hline & 8 & 36 & 32 & 27 & 2.51 & Medial Frontal Gyrus (BA 9) \\
\hline \multirow[t]{3}{*}{ Pictures } & 36 & 48 & -8 & 121 & 2.89 & Middle Frontal Gyrus (BA 11) \\
\hline & -40 & 50 & 4 & 81 & 2.59 & Middle Frontal Gyrus (BA 10) \\
\hline & -38 & 34 & 36 & 67 & 2.83 & Middle Frontal Gyrus (BA 9) \\
\hline Cross-Modality & 18 & 40 & 28 & 27 & 2.54 & Medial Frontal Gyrus (BA 8/9) \\
\hline Same-Modality & 44 & 16 & -8 & 43 & 2.77 & Inferior Frontal Gyrus (BA 47) \\
\hline \multicolumn{7}{|l|}{ Temporal Gyri } \\
\hline \multirow[t]{3}{*}{ Pictures } & -62 & -40 & -8 & 96 & 2.83 & Middle Temporal Gyrus (BA 21) \\
\hline & 64 & -38 & -8 & 112 & 3.16 & Middle Temporal Gyrus (BA 21) \\
\hline & -50 & -40 & 20 & 17 & 2.44 & Superior Temporal Gyrus (BA 22) \\
\hline Cross-Modality & -44 & -26 & -4 & 52 & 3.02 & Middle Temporal Gyrus (BA 21) \\
\hline \multirow[t]{3}{*}{ Same-Modality } & -44 & 10 & -12 & 62 & 2.69 & Superior Temporal Gyrus (BA 38) \\
\hline & -64 & -16 & -8 & 442 & 2.84 & Middle Temporal Gyrus (BA 21) \\
\hline & 64 & -18 & -4 & 18 & 2.40 & Middle Temporal Gyrus (BA 21) \\
\hline \multicolumn{7}{|l|}{ Insula } \\
\hline \multirow[t]{2}{*}{ Pictures } & 42 & 8 & -4 & 87 & 2.67 & Insula (BA 13) \\
\hline & -38 & 18 & 8 & 65 & 3.06 & Insula (BA 13) \\
\hline Cross-Modality & 44 & -2 & 8 & 338 & 3.09 & Insula (BA 13) \\
\hline \multirow[t]{2}{*}{ Same-Modality } & 42 & -8 & 8 & 39 & 2.45 & Insula (BA 13) \\
\hline & 32 & -20 & 12 & 31 & 2.38 & Insula \\
\hline \multicolumn{7}{|c|}{ Cuneus/Precuneus } \\
\hline Sentences & 12 & -68 & 12 & 120 & 3.08 & Cuneus (BA 17) \\
\hline \multirow[t]{2}{*}{ Pictures } & -10 & -70 & 28 & 49 & 2.80 & Precuneus (BA 7) \\
\hline & 14 & -68 & 28 & 37 & 2.49 & Precuneus (BA 7) \\
\hline \multicolumn{7}{|l|}{ Cerebellum } \\
\hline Pictures & 22 & -88 & -24 & 38 & 2.57 & Cerebellum \\
\hline \multicolumn{7}{|c|}{ Lingual/Fusiform Gyrus } \\
\hline \multirow[t]{3}{*}{ Cross-Modality } & 12 & -98 & -12 & 219 & 2.88 & Lingual Gyrus (BA 18) \\
\hline & 14 & -78 & -4 & 25 & 2.46 & Lingual Gyrus (BA 18) \\
\hline & 26 & -92 & -12 & 20 & 2.38 & Fusiform Gyrus (BA 18) \\
\hline \multicolumn{7}{|l|}{ Precentral Gyrus } \\
\hline Cross-Modality & -50 & 2 & 12 & 150 & 2.92 & Precentral Gyrus (BA 4) \\
\hline \multicolumn{7}{|l|}{ Cingulate Gyrus } \\
\hline \multirow[t]{2}{*}{ Same-Modality } & 22 & -44 & 24 & 93 & 2.80 & Cingulate Gyrus (BA 23) \\
\hline & 18 & 26 & 32 & 16 & 2.52 & Cingulate Gyrus (BA 32) \\
\hline
\end{tabular}


relatively stable "individual difference" variable expressed at the level of site-specific neuronal activity. T he results reported here are compatible with either alternative. At any rate, it is possible that relatively stable "structural" differences in blood flow, and hence neuronal activity, may exist. Further exploration of this issue is clearly called for, to enhance our understanding of the role of M TL in memory, as well as that of other brain regions.

\section{MEMORY PROCESSES IN MTL AND BEYOND}

Every one of the four studies yielded at least one brain/behavior correlation site in M TL, although in one case we had to lower the predetermined threshold a bit to pick up the correlation. Interestingly, all these MTL correlations were positive. The observation that activity in $M T L$ regions correlated positively with recognition memory performance has implications for the interpretation of their functional role. O ne general point is that such data indicate that it is not simply the attempt to remember that activates M T L regions. Rather these regions seem to play a special role in successful recognition. This may be related to findings that the degree of activation of $M T L$ regions during encoding is related to subsequent memory performance (Fernandez et al., 1998; Brewer et al., 1998; Wagner et al., 1998; C ahill and M CG augh, 1998). It is still an open question what specific aspect of successful recognition is related to $M T L$ activation, but we note that our findings are in keeping with proposals that $M T L$ activation is related to the experience of conscious recollection (Schacter et al., 1996; H eckers et al., 1998).

$M$ any other sites of correlations, both positive and negative, were observed in brain regions that have become quite familiar from the many neuroimaging studies of memory that have used subtraction techniques as well as correlational analyses (for reviews, see Buckner and Tulving, 1995; Cabeza and Nyberg, 1997). W ith regard to consistent findings across studies, positive correlations were observed in the cuneus/precuneus region. This agrees with previous correlational results (Grasby et al., 1993a). $M$ oreover, early data from subtraction analyses associated this region with episodic retrieval (G rasby et al., 1993b; Fletcher et al., 1995b), although its functional role was not clear. Subsequent work has shed some light on its role (Fletcher et al., 1997). A particularly interesting finding in the present context is that of higher activation in precuneus during conditions of higher compared to lower levels of episodic retrieval (Kapur et al., 1995b). Positive correlations were also found across studies in fusiform gyrus and premotor cortex. Based on what we know from subtraction analyses these regions do not stand out as episodic retrieval regions (C abeza and N yberg, 1997), but it is noteworthy that the significant correlation between verbal episodic memory and glucose utilization in left M TL reported by D esgranges et al (1998b) implicated the fusiform gyrus. N egative correlations were observed in bilateral frontal, temporal, and insular regions. Thus, subjects who performed well tended to have lower activity in these regions compared to subjects who recognized fewer items.
Although we cannot as yet say much about the "meaning" of positive and negative correlations, it seems reasonable to make something out of what looks like a spatial pattern, or a possible pattern, when we examine the sites of positive and negative activations in the various (large) anatomical regions of the brain, as shown in Tables 2 and 3: T here were no positive correlations in two large brain areas, namely (lateral) temporal gyri and insula, in which our exercise revealed negative correlations. These regions have frequently shown up in other PET studies of memory (G rasby et al., 1994; Tulving et al., 1994b,c; Fink et al., 1996; C abeza et al., 1997; Ghaem et al., 1997), and are known to be almost always damaged in anterograde amnesia (K apur et al., 1994).

$D$ oes this suggest that the brain will turn out to have regions in which only positive memory-based correlations with neural activity are found, and others in which there are only negative correlations? It is far too early to call the outcome; the future will tell. We must also remember that the spatial pattern is created by both the presences and absences of correlations, and the problem with absences is that in neuroimaging studies they are largely uninterpretable (Buckner and Tulving, 1995). Everybody knows that the easiest way to "produce" a lack of activation is to do a study with a small sample of subjects (Andreasen et al., 1996).

The role of the frontal lobes in episodic memory is complex, and subtraction analyses have revealed positive, negative, or no association between degree of prefrontal activity and memory performance (for a recent review, see Gabrieli et al., 1997). Of special interest here are findings of lower prefrontal activity during higher levels of retrieval. Such a pattern was observed by Schacter et al. (1996) and interpreted in terms of retrieval effort. Similarly, in the present analyses, those subjects who scored low may have tried harder to recognize the stimuli than those performing well. With regard to lower activity in various temporal and insular regions, it can be noted that inhibition of activity in temporal regions has been suggested to be important for episodic retrieval (N yberg et al., 1996c). M oreover, Cabeza et al. (1997) found a negative correlation between insular activity during encoding and performance on a delayed test of episodic memory, and they suggested that insular activation may be disruptive rather than beneficial.

M ore generally, although these correlational findings have been discussed region by region, we like to stress the possibility that several of the involved regions, showing positive as well as negative correlations, are components of the samenetwork of brain regions that mediates successful episodic retrieval. In such a network, increased as well as decreased regional activity are equally important for a successful outcome (e.g., recognition of an item). Such a "success network" probably operates in conjunction with a more general episodic retrieval network (N yberg et al., 1996b).

\section{"WHAT" AND "HOW" MEMORY SITES}

$M$ any previous "subtraction" studies that we briefly reviewed in the introduction of this report have provided suggestive evidence 
of the existence of brain sites that are differentially active in two comparison tasks. The results from the covariance analyses we have presented here have provided suggestive evidence of the existence of brain sites whose activity in different subjects is correlated with the "goodness" of the subjects' cognitive/ behavioral performance. We refer to the activation sites yielded by subtraction analyses as the "what" sites: their activity "reveals" (i.e., is correlated with) what the system is doing, or what kind of a task it is engaged in. And we refer to the activation sites yielded by brain/cognition covariance analyses as the "how" sites: their activity "reveals" (i.e., is correlated with) how well the system is performing a given task.

Given the suggestive evidence of the existence of these two kinds of memory-related activation sites in the brain, it makes sense to ask how the two are related. Are the "what" sites the same as the "how" sites? Always? Or are the "how" sites in a given task a subset of the "what" sites of the same task?

It is quite possible that the "what" regions that distinguish between the presence and the absence of a process, or that are differentially "involved" in two tasks, are also "how" regions whose activity is associated with how well a given single task is carried out. It is also possible, of course, that the "what" and the "how" of a task are subserved by largely separate regions. Because of the novelty of the distinction and near-total absence of any relevant data, there is little that one can say definitively on the topic at this time. We are dealing with an empirical question here that only future research can answer. This future research will not only assess the validity of the distinction between the hypothetical "what" and "how" regions, but will also clarify the relation between them.

It is useful to firmly keep in mind one purely logical difference between "what" and "how" regions: "W hat" regions are always and necessarily associated with the difference between two tasks. The locations of "what" sites, therefore, can vary with the task of interest (the target task) as well as with the control task (the reference task). "H ow" regions, on the other hand, are associated with a given single task, and their locations are not directly affected by any other task. For this reason alone one should not expect a complete overlap between "what" and "how" memory sites.

The distinction between "what" and "how" memory regions of the brain can be seen as related to the findings of some empirical evidence from our previous studies. In two experiments we found evidence that some brain regions, most notably a right prefrontal region, are generally involved in episodic memory retrieval, whereas more posterior regions seem to be specifically activated during conditions of higher levels of retrieval performance (K apur et al., 1995b; Nyberg et al., 1995). Thus the right prefrontal region may be considered a "what" region and specific posterior (and M TL) regions "how" regions. Similarly, in a study of retrieval of item, spatial, and temporal information (N yberg et al., 1996b), we found that several regions, including right anterior prefrontal cortex, were involved in retrieval regardless of type of information, whereas other frontal (and temporal) regions were activated depending on the to-be-retrieved information (item, spatial, or temporal information). We described this pattern of results in terms of "general" and "specific" brain regions mediating various aspects of episodic retrieval, but the general/specific distinction could be mapped onto the distinction between "what" and "how."

For what it is worth, we notehere that the five M TL "how" sites listed in Table 2 are localized more-or-less evenly along the rostrocaudal axis of the hippocampus, without showing any tendency to conform to the HIPER pattern. This observation suggests that there need not be indeed any tight coupling between the "what" and the "how" regions. As we mentioned earlier, our first ventures into correlational PET analyses (Nyberg et al., 1996d) also yielded "how" sites in the rostral part of M TL, thus further adding to the separability of the localization of the two kinds of memory-related regions. It is difficult, of course, to draw even tentative conclusions on the basis of data as sparse as those available now. This is why our speculations about the relation between "what" and "how" regions primarily serve the function of guiding future research.

\section{UNILATERAL MTL CORRELATIONS?}

Another interesting feature of the MTL "how" sites listed in Table 2 is that they are unilateral, that is, found only in one hemisphere in any given study. Because of the small number of observations involved, it would be difficult to assign much significance to the fact they are "mostly" in the left hemisphere. $\mathrm{N}$ evertheless, the same tendency for M TL activations to occur unilaterally is also found in meta-analyses-based PET rCBF (Lepage et al., 1998) or FM RI BO LD (Schacter and Wagner, this issue) studies. In these analyses the data are more convincing, because the sample sizes are larger.

It is important to note that the observation of (mostly) left-lateralized MTL activations in the data set summarized in Table 2 is not, for whatever reason, a necessary outcome of the method of analysis as such. Such necessity is contraindicated by the data in Table 3, which lists the sites of negative correlations. There are five pairs of bilateral activations: two in the frontal gyri (sentences and pictures), two in temporal gyri (pictures and same-modality), and one in cuneus/precuneus (pictures). Similar bilateral activations of a given small region are frequently observed in PET studies of memory.

As is the case with most PET findings at the present early stage of research, the apparent unilaterality of M TL activations has no ready physiological explanation. The major reason for the difficulty lies in the fact that much of previous research on hippocampal memory has dealt with the hippocampus bilaterally and has glossed over the possibility of its unilateral functioning. In lesion work with nonhuman animals, lesions are usually bilateral. In studies of human patients with M TL damage, laterality as an issue has usually only risen in connection with the traditional orientation in terms of material specificity - right, verbal stimuli; left, nonverbal. Although the language-based laterality differences cannot be excluded, they do not account for the total pattern. 


\section{MEANING AND "REALITY" OF THE FINDINGS}

As one contemplates the data reported here, as well as many other data reported in other articles in the Special Issue, and does so against the backdrop of a rapidly growing rich literature on neurocognition of memory, it is easy to feel satisfied with the pace of progress. Even if we still do not have any good ideas about the role of "the hippocampus" in memory, at least it looks as if we are collecting data about memory processes in living brains of the kind that one could only have dreamt about as little as 10 years ago.

Before we get carried away by the exuberant enthusiasm that frequently affects players of a new game, and in the interests of serious science, we should ask a simple question: H ow "real" are these findings? We raise this question because we believe it is important. Although the problem of reality of findings has been noted and discussed before (Eustache et al., 1995; D esgranges et al., 1998a), it frequently tends to be overlooked.

The problem is one of false-positives: artifactual activations that appear in the data because of the procedure used rather than because of the physiology of cognition. We know that a certain proportion of activations are artifactual. What we do not know is how many and which. In our own studies it must be the case that some vectors in a huge array of nearly 60,000 such, each representing the blood flow counts of the $\mathrm{N}$ subjects in a given voxel in the Talairach space, would be positively or negatively correlated with some other vectors, including those comprising entirely randomly selected numbers.

This simple fact means that it is not sufficient to identify "real" (biologically relevant) correlation sites by statistical significance levels alone. Finding and reporting of single unreplicated correlations in and of itself does not carry much weight. Such a procedure would lead to the inclusion of many false-positives among the "real" brain/mind correlations. To guard against such errors, and to minimize their occurrence, one must rely heavily on consistency analysis. The difference between real and artifactual activation sites - both "what" and "how" - lies in their replication across studies: Specific real activations replicate, spurious ones do not. In our studies, both those done previously (Nyberg et al., 1996d) and those reported here, we have been fully aware of the difficulty and have used consistency and replicability of activation sites as an important criterion in reporting the findings.

Even full awareness of the possibility of false-positive errors and consequent insistence on consistency, however, cannot guarantee that every single one of the correlation sites that we report here is "real." There is little doubt that an unknown proportion of them are spurious. And there is no way to tell, from any single study, which ones they are. It is only painstaking replicative research that will eventually sort out the wheat from the chaff.

The same logic, of course, applies to both the correlational and the subtractive methods. In subtraction studies, too, it is almost al ways the case that some observed "activations" represent nothing more than noise, and again it is not possible to establish their identity on the basis of a single study.
These simple logical facts urge care in the design of the studies, and caution in the interpretation of findings. It isfor these reasons that larger systematic patterns of activation or correlations yielded by neuroimaging studies, such as HERA in the frontal lobes (Tulving et al., 1994a; Nyberg et al., 1996a; Grady, 1999) and HIPER in MTL (Lepage et al., 1998; Schacter and Wagner, 1999), are going to be more informative in the long run than are the results of individual studies: They cannot be produced by random factors.

\section{CONCLUSIONS}

The story we have told here serves more to illustrate what PET and FM RI can contribute to the understanding of memory in the brain than it does to describe what has al ready been accomplished. We are still at the very early stages of the functional neuroimaging game and are still learning how to play it properly. In doing so, we must remember that the medial temporal lobes and the hippocampus, and their role in memory, did not evolve for the convenience of cognitive neuroscientists.

The purpose of this work was to explore the usefulness of the brain/behavior correlational method in the study of the role of MTL and other brain regions in memory. We wish to emphasize both "M TL" and "other regions" because it is clear that a final understanding of the role of MTL in memory does not only require knowledge of the inner workings of various individual M TL compartments and their relatedness, but also that of their mutual interactions with other memory-related cerebral regions, in keeping with the spirit of memory networks in "the hippocampus and beyond" (Tulving and M arkowitsch, 1997). Functional neuroimaging, like any other single technique that scientists use, is in many ways imperfect.

The meta-analysis of across-subjects correlations betwen regional cerebral blood flow and recognition-memory performance that we reported here shows that neuronal activity in specific regions in the brain (the "how" sites), including those in and near the hippocampus, covaries systematically with the accuracy with which different individuals perform recognition tasks. O ne possible interpretation of our findings, to betaken with a big grain of salt, is that regions exist in "memory areas" of the brain whose level of neuronal activity determines how well the individual performs on the task. These brain/cognition correlational data complement data from studies using subtraction analyses, data that point to the regions (the "what" sites) that are systematically involved in mediating the execution of a given task with its specific processing requirements.

We think of the data we have reported here as representing another tiny step toward the ultimate goal of functional neuroimaging - that of mapping of brain activity, in and beyond MTL regions, into mental activity, and vice versa. H owever, because we still know rather little about the validity and reliability of neuroimaging data, we must wait for future research to tell us how much progress the "tiny" step represents. 


\section{REFERENCES}

Aggleton JP, Brown M W. Episodic memory, amnesia, and the hippocampal-anterior thalamic axis. Behav Brain Sci (in press).

Aggleton JP, Saunders RC. 1997. The relationships between temporal lobe and diencephalic structures implicated in anterograde amnesia. Memory 5:49-71.

Andreasen N C, Arndt S, C izadlo T, O 'Leary DS, Watkins G L, Ponto LL, Hichwa RD . 1996. Sample size and statistical power in [150 ]H 20 studies of human cognition. J Cereb Blood Flow M etab 16:804-816.

Baxendale SA, Van Paesschen W, Thompson PJ , Connelly A, D uncan JS, $H$ arknessW F, Shorvon SD . 1998. The relationship between quantitative MRI and neuropsychological functioning in temporal lobe epilepsy. Epilepsia 39:158-166.

Berthoz A. 1997. Parietal and hippocampal contribution to topokinetic and topographic memory. Philos Trans R Soc Lond [Biol] 352:14371448.

Brewer JB, Zhao Z, Desmond JE, Glover GH, Gabrieli JD. 1998. $M$ aking memories: brain activity that predicts how well visual experience will be remembered. Science 281:1185-1187.

Buckner RL, Tulving E. 1995. N euroimaging studies of memory: theory and recent PET results. In: Boller F, Grafman J, editors. $\mathrm{H}$ andbook of neuropsychology. Amsterdam: Elsevier. p. 439-466.

Cabeza R, N yberg L. 1997. Imaging cognition: An empirical review of PET studies with normal subjects. J Cognit N eurosci 9:1-26.

Cabeza R, Grady CL, N yberg L, M clntosh AR, Tulving E, Kapur S, Jennings J M , H oule S, Craik FIM . 1997. Age-related differences in neural activity during memory encoding and retrieval: a positron emission tomography study. J N eurosci 17:391-400.

Cahill L, McGaugh JL. 1998. M echanisms of emotional arousal and lasting declarative memory. Trends N eurosci 21:294-299.

Desgranges B, Baron JC, Eustache F. 1998a. The functional neuroanatomy of episodic memory: The role of the frontal lobes, the hippocampal formation, and other areas. N euroimage 8:198-213.

D esgranges B, Baron JC, de La Sayette V, Petit-Taboué M C, Lechevalier B, Eustache F. 1998b. The neural substrates of memory systems in Alzheimer's disease: A PET study of resting brain glucose utilization. Brain 121:611-631.

Dobbins IG, Kroll NE, Tulving E, Knight RT, Gazzaniga M S. 1998. Unilateral medial temporal lobe memory impairment: type deficit, function deficit, or both? N europsychologia 36:115-127.

Dolan RJ, Fletcher PC. 1997. D issociating prefrontal and hippocampal function in episodic memory encoding. N ature 388:582-585.

Epstein R, Kanwisher N. 1998. A cortical representation of the local visual environment. N ature 392:598-601.

Eustache F, Rioux P, D esgranges B, M archal G, Petit-Taboue M C, D ary M, Lechevalier B, Baron JC. 1995. Healthy aging, memory subsystems and regional cerebral oxygen consumption. N europsychologia 33:867-887.

Fernandez G, Weyerts $H$, Schrader-Bolsche $M$, Tendolkar I, Smid H GO M , Templemann C, H inrichs H, Scheich H , Elger CE, M angun GR, H einze H-J. 1998. Succesfful verbal encoding into episodic memory engages the posterior hippocampus: a parametrically analyzed functional magnetic resonance imaging study. J Neurosci 18:1841-1847.

Fink GR, M arkowitsch HJ, Reinkemeier M, Bruckbauer T, Kessler J, H eiss W -D . 1996. Cerebral representation of one's own past: N eural networks involved in autobiographical memory. J N eurosci 16:42754282.

Fletcher PC, Frith CD, Baker SC, ShalliceT, Frackowiak RSJ, D olan RJ. 1995a. The mind's eye - precuneus activation in memory-related imagery. N euroimage 2:195-200.

Fletcher PC, Frith CD, Grasby PM, Shallice T, Frackowiak RSI, D olan RJ. 1995b. Brain systems for encoding and retrieval of auditory-verbal memory: an in vivo study in humans. Brain 118:401-416.
Fletcher PC, Frith CD, Rugg M D . 1997. The functional neuroanatomy of episodic memory. Trends N eurosci 20:213-218.

Friston KJ, H olmes AP, Worsley KJ, Poline J-P, Frith CD, Frackowiak RS). 1995. Statistical parametric maps in functional imaging: a general linear approach. H um Brain M ap 2:189-210.

Fujii T, O kuda J, Kawashima R, Yamadori A, Fukatsu R, Suzuki K, Ito M, G oto R, Fukuda H. 1997. Different roles of the left and right parahippocampal regions in verbal recognition: a PET study. N euroreport 8:1113-1117.

Gabrieli JDE, Brewer JB, Desmond JE, Glover GH. 1997. Separate neural bases of two fundamental memory processes in the human medial temporal lobe. Science 276:264-266.

G haem O, M ellet E, C rivello F, Tzourio N, M azoyer B, Berthoz A, D enis M. 1997. M ental navigation along memorized routes activates the hippocampus, precuneus, and insula. N euroreport 8:739-744.

Grady C. 1999. N euroimaging and activation of the frontal lobes. In: $M$ iller BL, Cummings J L, editors. The human frontal lobes: function and disorders. N ew York: Guilford Press. pp. 196-230.

Grasby PM , Frith CD, Friston KJ, Bench C, Frackowiak RSJ, D olan RJ. 1993a. Functional mapping of brain areas implicated in auditoryverbal memory function. Brain 116:1-20.

Grasby PM, Frith CD, Friston KJ, Frackowiak RSJ, D olan RJ. 1993b. Activation of the human hippocampal formation during auditoryverbal long-term memory function. N eurosci Lett 163:185-188.

Grasby PM , Frith CD, Friston KJ, Simpson J, Fletcher PC, Frackowiak RSJ, Dolan RJ. 1994. A graded task approach to the functional mapping of brain areas implicated in auditory-verbal memory. Brain 117:1271-1282.

Grunwald T, Lehnertz K, H einze H-J, H elmstaedter C, Elger CE. 1998. Verbal novelty detection within the human hippocampus proper. Proc N atl Acad Sci U SA 95:3193-3197.

H axby JV, Ungerleider LG, H orwitz B, M aisog J M , Rapoport SI, G rady $C L$. 1996. Face encoding and recognition in the human brain. Proc $N$ atl Acad Sci USA 93:922-927.

H eckers S, Rauch SL, G off D, Savage CR, Schacter D L, Fischman AJ, Alpert N M . 1998. Impaired recruitment of the hippocampus during conscious recollection in schizophrenia. N ature 1:318-323.

H elmstaedter C, G runwald T, Lehnertz K, G leissner U, Elger CE. 1997. Differential involvement of left temporolateral and temporomesial structures in verbal declarativelearning and memory: evidence $>$ from temporal lobe epilepsy. Brain Cognition 35:110-131.

Henke K, Buck A, W eber B, Wieser H G. 1997. H uman hippocampus establishes associations in memory. $\mathrm{H}$ ippocampus 7:249-256.

Kapur N, Barker S, Burrows EH, Ellison D, Brice J, Illis LS, Scholey K, Colbourn K, W ilson B, Loates M . 1994. H erpes simplex encephalitis: Long term magnetic resonance imaging and neuropsychological profile. J Neurol N eurosurg Psychiatry 57:1334-1342.

Kapur N, Friston KJ, Young A, Frith CD, Frackowiak RSJ. 1995. Activation of human hippocampal formation during memory for faces: a PET study. Cortex 31:99-108.

Kapur S, Craik FIM, Jones C, Brown GM , H oule S, Tulving E. 1995. Functional role of the prefrontal cortex in retrieval of memories: a PET study. N euroreport 6:1880-1884.

Kelley W M . 1998. Hemispheric specialization in human dorsal frontal cortex and medial temporal lobe for verbal and nonverbal memory encoding. N euron 20:927-936.

Knight RT. 1996. Contribution of human hippocampal region to novelty detection. N ature 383:256-259.

Kopelman M D, Stevens TG, Foli S, G rasby P. 1998. PET activation of the medial temporal lobe in learning. Brain 121:875-887.

Lepage M, H abib R, Tulving E. 1998. H ippocampal PET activations of memory encoding and retrieval: the HIPER model. H ippocampus 8:313-322.

M aguire EA. 1997. H ippocampal involvement in human topographical memory: evidence from functional imaging. Philos Trans R Soc L ond [Biol] 352:1475-1480. 
M aguire EA, Burke T, Phillips J, Staunton H. 1996a. Topographical disorientation following unilateral temporal lobe lesions in humans. N europsychologia 34:993-1001.

M aguire EA, Frackowiak RSJ, Frith CD. 1996b. Learning to find your way: a role for the human hippocampal formation. Proc R Soc Lond [Biol] 263:1745-1750.

Maguire EA, Frith CD, Burgess N, Donnett JG, O'Keefe J. 1998. Knowing where things are parahippocampal involvement in encoding object locations in virtual large-scale space. J Cognit Neurosci 10:61-76.

M artin A, W iggs CL, Weisberg J. 1997. M odulation of human medial temporal lobe activity by from meaning, and experience. $\mathrm{H}$ ippocampus 7:587-593.

M attioli F, Grassi F, Perani D, Cappa SF, M iozza A, Fazio F. 1996. Persistent post-traumatic retrograde amnesia: a neuropsychological and (18F)FD G PET study. Cortex 32:121-129.

M clntosh AR, Bookstein FL, H axby JV, Grady CL. 1996. Spatial pattern analysis of functional brain images using partial least squares. Neuroimage 3:143-157.

M ilner B, Johnsrude I, Crane J. 1997. Right medial temporal-lobe contribution to object-location memory. Philos Trans R Soc Lond [Biol] 352:1469-1474.

N yberg L, Tulving E, H abib R, N ilsson L-G, Kapur S, H oule S, Cabeza $R, M$ clntosh AR. 1995. Functional brain maps of retrieval mode and recovery of episodic information. N euroreport 7:249-252.

N yberg L, Cabeza R, Tulving E. 1996a. PET studies of encoding and retrieval: the HERA model. Psychonomic Bull Rev 3:135-148.

N yberg L, M clntosh AR, Cabeza R, H abib R, H oule S, Tulving E. 1996b. General and specific brain regions involved in encoding and retrieval of events: what, where, and when. Proc N atl Acad Sci U SA 93:11280-11285.

N yberg L, M clntosh AR, Cabeza R, N ilsson L-G, H oule S, H abib R, Tulving E. 1996c. N etwork analysis of positron emission tomography regional cerebral blood flow data: Ensemble inhibition during episodic memory retrieval. J N eurosci 16:3753-3759.

N yberg L, M clntosh AR, Houle S, Nilsson L-G, Tulving E. 1996d. Activation of medial temporal structures during episodic memory retrieval. N ature 380:715-717.

Parkin AJ. 1997. H uman memory: novelty, association and the brain. Curr O pin N eurobiol 7:R 768-R 769.

Petersson KM , Elfgren C, Ingvar M . 1997. A dynamic role of the medial temporal lobe during retrieval of declarative memory in man. N euroimage 6:1-11.

Ridley RM, Baker HF. 1997. Evidence for a specific information processing deficit in monkeys with lesions of the septo-hippocampal system. Cortex 33:167-176.

Rugg M D, Fletcher PC, Frith CD, Frackowiak RSJ, Dolan RJ. 1997. Brain regions supporting intentional and incidental memory: a PET study. N euroreport 8:1283-1287.

Schacter D L. 1997. The cognitive neuroscience of memory: perspectives from neuroimaging research. Philos Trans R Soc Lond [Biol] 352: 1689-1695.
Schacter DL, Wagner AD. 1999. M edial temporal lobe activations in fM RI and PET studies of episodic encoding and retrieval. H ippocampus 9.

Schacter DL, Reiman E, U ecker A, Polster M R, Yun LS, Cooper LA. 1995. Brain regions associated with retrieval of structurally coherent visual information. N ature 376:587-590.

Schacter DL, Alpert N M, Savage CR, Rauch SL, Albert MS. 1996. Conscious recollection and the human hippocampal formation: Evidence from positron emission tomography. Proc Natl Acad Sci USA 93:321-325.

Schacter D L, U ecker A, Reiman E, Yun LS, Bandy D, Chen K, Cooper LA, Curran T. 1997. Effects of size and orientation change on hippocampal activation during episodic recognition: a PET study. N euroreport 8:3993-3998.

Schreurs BG, M clntosh AR, Bahro M, Herscovitch P, Sunderland T, Molchan SE. 1997. Lateralization and behavioral correlation of changes in regional cerebral blood flow with classical conditioning of the human eyeblink response. J N europhysiol 77:2153-2163.

Squire LR. 1992. Memory and the hippocampus: a synthesis from findings with rats, monkeys, and humans. Psychol Rev 99:195-231.

Squire LR, O jemann JG, M iezin FM, Petersen SE, Videen TO, Raichle ME. 1992. Activation of the hippocampus in normal humans: $A$ functional anatomical study of memory. Proc Natl Acad Sci USA 89:1837-1841.

Stern CE, C orkin S, G onzalez RG, Guimaraes AR, Baker JR, Jennings PJ, Carr CA, Sugiura RB, Vedantham V, Rosen BR. 1996. Thehippocampal formation participates in novel picture encoding: Evidence from functional magnetic resonance imaging. Proc Natl Acad Sci USA 93:8660-8665.

Talairach J, Tournoux P. 1998. Co-planar stereotaxic atlas of the human brain. N ew York: Thieme M edial Publishers, Inc.

Tulving E. 1983. Elements of episodic memory. New York: Oxford University Press.

Tulving E, M arkowitsch H J. 1997. M emory beyond the hippocampus. Curr 0 pin N eurobiol 7:209-216.

Tulving E, Kapur S, Craik FIM, Moscovitch M, Houle S. 1994a. $\mathrm{H}$ emispheric encoding/retrieval asymmetry in episodic memory: positron emission tomography findings. Proc Natl Acad Sci USA 91:2016-2020.

Tulving E, Kapur S, M arkowitsch HJ, Craik FIM, H abib R, H oule S. 1994b. N euroanatomical correlates of retrieval in episodic memory: auditory sentence recognition. Proc Natl Acad Sci USA 91:20122015.

Tulving E, M arkowitsch H J, Kapur S, H abib R, H ouleS. 1994c. N ovelty encoding networks in the human brain: positron emission tomography data. N euroreport 5:2525-2528.

Tulving E, M arkowitsch HJ, Craik FIM, H abib R, H oule S. 1996. $\mathrm{N}$ ovelty and familiarity activations in PET studies of memory encoding and retrieval. Cereb Cortex 6:71-79.

Wagner $A D$, Schacter $D L$, Rotte $M$, Koutstaal W, M aril A, Dale AM, Rosen BR, Buckner RL. 1998. Building memories: Remembering and forgetting of verbal experiences as predicted by brain activity. Science 281:1188-1191. 